\title{
Civilisations
}

Revue internationale d'anthropologie et de sciences

humaines

$57 \mid 2008$

Tourisme, mobilités et altérités contemporaines

\section{Spatialités et territorialités du tourisme}

Dialectique du flux et de l'ancrage dans les Alpes

Bernard Debarbieux, Cristina Del Biaggio et Mathieu Petite

\section{(2) OpenEdition}

\section{Journals}

Édition électronique

URL : http://journals.openedition.org/civilisations/1085

DOI : 10.4000/civilisations. 1085

ISSN : 2032-0442

Éditeur

Institut de sociologie de l'Université Libre de Bruxelles

Édition imprimée

Date de publication : 29 décembre 2008

Pagination : 75-89

ISBN : 2-87263-022-8

ISSN : 0009-8140

Référence électronique

Bernard Debarbieux, Cristina Del Biaggio et Mathieu Petite, "Spatialités et territorialités du tourisme », Civilisations [En ligne], 57 | 2008, mis en ligne le 29 décembre 2011, consulté le 19 avril 2019. URL

http://journals.openedition.org/civilisations/1085; DOI : 10.4000/civilisations.1085

(c) Tous droits réservés 


\title{
Spatialités et territorialités du tourisme \\ Dialectique du flux et de l'ancrage dans les Alpes
}

\section{Bernard DEBARBIEUX, Cristina DEL BIAGGIO, Mathieu PETITE}

\begin{abstract}
Résumé : Cet article propose un cadre général d'interprétation pour analyser le phénomène touristique dans un contexte - celui de la mondialisation - de mise en circulation généralisée des individus, des biens, de l'argent et des images. Il étudie plus particulièrement les stratégies déployées ici et là pour ancrer dans un lieu les flux divers qu'engendre la mobilité touristique, en montrant aussi que cet ancrage au lieu procède souvent d'une mobilisation d'échelles intermédiaires. Cette analyse dialectique, couplant logiques de circulation et d'ancrage, est appliquée aux Alpes, et plus précisément encore, à trois études de cas, le Grosses Walsertal (Autriche), le Val d'Hérens (Suisse) et Chamonix (France) où les stratégies d'ancrage se sont focalisées sur des éléments différents : les liens sociaux, le patrimoine, les valeurs communes.
\end{abstract}

Mots-clés : circulation, ancrage, échelles, territorialisation, Alpes, tourisme, patrimoine.

Abstract: This paper proposes a general frame for understanding tourism in the context of globalization. In this frame, tourism is conceptualized as a phenomenon simultaneously influenced (1) by the growing circulation of people, goods, money and images, that is by growing mobilities; and (2) by the will and the need frequently expressed in touristic places to root the touristic activity. The paper shows that strategies of territorialization of the touristic activities are often conceived at a local and at a "medium" level. After the general presentation of the conceptual frame, the second section of the paper provides illustration with the case of the Alps, through the description of the touristic strategies of three small Alpine regions, Grosses Walsertal (Autriche), Val d'Hérens (Suisse) and Chamonix (France). These case studies illustrate various ways of combining different strategies of territorialization.

Key words: circulation, rootedness, scales, territorialization, Alps, tourism, cultural heritage. 
$\mathrm{L}$

es sciences sociales ont fortement insisté sur l'idée que le tourisme met en présence des individus temporairement mobiles et des individus sédentaires (temporairement ou non) qui accueillent ou côtoient les premiers sur leur lieu de destination. De fait, au moment de son «invention", le tourisme a mis en mouvement des personnes qui l'étaient peu ou d'une autre façon et il a amené des générations de citadins dans des lieux où généralement la sédentarité dominait. C'est encore le cas aujourd'hui dans de très nombreux contextes, notamment quand les touristes occidentaux investissent des sites lointains habités par des populations dotées d'une moindre mobilité ou d'une mobilité de nature peu comparable. Appréhender le tourisme du point de vue de la différence de mobilité, couplé avec d'autres différences, sociales, économiques et culturelles, constitue donc assurément une piste intéressante dont la portée heuristique reste grande.

Toutefois, on ne peut ignorer que dans un nombre croissant de situations, cette différence s'est atténuée, quand elle n'a pas disparu. Quand des touristes du monde entier visitent Londres, New York ou Venise, ils côtoient sur place des gens parfois tout aussi mobiles qu'eux, eux aussi touristes à leurs heures. Quand des touristes se rendent dans les grandes stations littorales d'Europe ou d'Amérique du nord, ils ont affaire à des prestataires de service - hôteliers, commerçants, animateurs, etc. - qui ont souvent, mais à d'autres moments, des pratiques comparables. Autrement dit, s'il est devenu banal d'écrire que le monde occidental contemporain est marqué par des formes de mobilité généralisée (Urry 2005), les recherches sur le tourisme ne semblent pas encore en avoir tiré toutes les conséquences.

Il existe de très nombreuses manières de problématiser ces mobilités croisées. On proposera ici d'adopter un point de vue de géographe, ancré dans une problématique spatiale et territoriale. Une telle problématique articule la prise en compte de la spatialité, conçue comme l'agencement des phénomènes divers qui composent l'espace géographique, et celle de la territorialité, conçue comme l'expression d'une volonté délibérée de construire certains de ces agencements. Les agencements spatiaux et les construits territoriaux dont il sera question ici sont ceux qui participent et résultent de l'activité touristique elle-même. L'analyse mettra l'accent sur la tension qui existe entre les flux divers (de personnes, d'images, de biens, etc.) impliqués dans le phénomène touristique, et les stratégies territoriales d'ancrage adoptées par les populations locales pour réguler ces flux.

Cette façon de problématiser la spatialité et la territorialité, de coupler dans l'analyse circulation et ancrage, a des précédents nombreux en géographie depuis la fin du $19^{\mathrm{e}}$ siècle. Dans les années 1950, Jean Gottmann en a même fait la principale clef de lecture de sa géographie politique. À ses yeux, les États modernes se caractérisent par cette capacité à réguler à leur échelle les flux hétérogènes qui les traversent (Gottmann 1952), notamment à l'aide de ce qu'il appelait «l'iconographie », soit l'ensemble des dispositifs symboliques à même d'attacher les populations à leur territoire national. Dans les années 1970, un couplage similaire a été théorisé et mis en œuvre par une génération de géographes anglophones qui se sont servis des concepts de space et place. Le concept de space leur a permis d'appréhender l'espace comme un champ sillonné de flux quand le concept de place visait à rendre compte des processus par lesquels les individus et les collectifs construisaient des formes d'attachement affectif ou symbolique (Relph 1976; 
Tuan 1977). Plus récemment encore, le renouvellement des études régionales, dans le cadre notamment de ce que les anglophones appellent la new regional geography ${ }^{1}$, a relancé la réflexion en insistant sur le rôle des constructions intentionnelles, symboliques, identitaires et/ou politiques dans l'émergence et la stabilisation de dynamiques régionales. Sans détailler davantage les contributions respectives de ces courants et les différences qui les caractérisent, force est de constater qu'une approche dialectique de ce que l'on propose d'appeler ici la circulation et l'ancrage participe d'un questionnement géographique déjà ancien. Ceci dit, on doit aussi constater que ce dernier n'a pas été véritablement appliqué au tourisme. Notre article, qui se veut général et synthétique, se place dans cette perspective et, en privilégiant un niveau local d'analyse, il s'intéresse à une région d'Europe, les Alpes, caractérisée par des pratiques touristiques intenses et anciennes. Plus précisément, il se focalise sur trois petites régions alpines ayant développé des stratégies d'ancrage différentes.

\section{Le tourisme et ses flux}

De quels flux et de quelles circulations parle-t-on exactement? À défaut de s'intéresser à toutes les formes de flux possibles, on s'arrêtera sur celles qui semblent avoir le plus d'implications dans les pratiques de territorialisation auxquelles cet article s'intéresse : la circulation des images et des modèles, les flux de biens et d'argent, et les déplacements de personnes.

Ces flux et leurs implications territoriales ont fait l'objet d'une attention particulière de la littérature académique de ces dernières années. Les nombreux auteurs qui se sont intéressés à la mondialisation, en prenant en compte la multiplicité de ses différentes formes - économique, informationnelle et culturelle notamment -, ont bien montré que ces différents flux avaient des effets combinés. Ils engendrent de nouveaux rapports à l'espace (Appadurai 1996; Featherstone, Lash, Robertson 1995; Offner, Pumain 1996), des formes renouvelées d'identité locale et régionale (Castells 1999 [1996]; Hannerz 1996) et de nouvelles territorialités (Raffestin 2006; Tarrius 2000). Dans cet article, on propose de retenir trois grands types de flux dont on pense qu'ils jouent un rôle décisif dans la construction spatiale et territoriale de l'activité touristique et de ses effets périphériques.

- Les flux d'images et de modèles d'abord, ont fortement marqué l'évolution des attentes et des offres touristiques. Le tourisme alpin repose sur un nombre certes croissant de modèles culturels, mais ceux-ci restent peu nombreux. Les représentations qui ont prévalu aux origines de ce tourisme - singularité du milieu naturel, esthétique paysagère, étrangeté des sociétés perçues comme traditionnelles et proches des rythmes de la nature (Reichler, Ruffieux 2000; Tissot 2000), préoccupations de santé aussi subsistent aujourd'hui. D'autres représentations s'y sont ajoutées, ouvrant le vaste champ des pratiques sportives et kinesthésiques (alpinisme, ski et spéléologie d'abord, parapente et canyoning plus récemment). Ces représentations et ces pratiques, dont plusieurs ont été inventées dans les Alpes elles-mêmes, se sont déployées à l'échelle de la chaîne toute entière, et même pour beaucoup à l'échelle de très nombreuses chaînes de montagnes à travers le monde (Debarbieux 1997). Dans beaucoup de régions de montagne, on a assisté à l'adoption de modèles d'équipement et d'aménagement très similaires, notamment dans et autour des stations de ski.

1. Pour une présentation et une analyse critique, voir Bradshaw 1990; Holmén 1995. 
- Les flux de biens et d'argent ensuite, constituent une dimension bien connue de la mobilité touristique. Activité de consommation, le tourisme suppose l'accès à des biens et des services via des transactions monétaires. Le propre des biens échangés dans ces circonstances est de disposer d'une valeur symbolique importante, liée aux modèles culturels auxquels les pratiques touristiques les rattachent. Les pratiques gastronomiques, le commerce d'images (photos, affiches, cartes postales, etc.) et des produits de l'artisanat participent de l'expérience touristique. Pris dans leur ensemble, les transferts d'argent liés à l'ensemble de ces transactions sont considérables, au point de contribuer fortement, voire majoritairement, à l'activité économique des régions alpines. L'adoption d'une monnaie unique, l'euro, dans la plupart des pays alpins, a contribué à fluidifier encore la circulation monétaire. Elle a aussi contribué à généraliser l'évaluation marchande des prestations, lesquelles, une fois monétarisées et devenues commensurables, sont de plus en plus systématiquement rapportées les unes aux autres. La montée en puissance somme toute assez récente des grands opérateurs, dans l'immobilier et les remontées mécaniques principalement, tend aussi à faire primer des logiques de rentabilité financière des investissements sur toute autre considération.

- La mobilité des personnes enfin, par laquelle se définit avant tout le tourisme. On évalue ainsi à 500 millions par an le nombre de nuitées passées dans les Alpes à des fins touristiques, ce qui en fait une des plus fortes concentrations de ce type dans le monde (Bätzing 2003). Par ailleurs, comme dans la plupart des régions touristiques d'Europe, les populations résidentes sont elles-mêmes promptes à se muer en touristes. Cette mobilité est proportionnellement plus forte encore dans les principales stations touristiques, puisqu'à la circulation touristique s'ajoute celle liée au caractère saisonnier et aux parcours professionnels des prestataires de services. Dans les Alpes, comme dans beaucoup d'autres régions, on a donc affaire à des mobilités croisées qui, d'une certaine façon, banalisent les pratiques des visiteurs aux yeux des résidents.

\section{Stratégies d'ancrage}

Si l'enchevêtrement de ces différents flux est inhérent au phénomène touristique, il porte aussi en lui plusieurs risques: banalisation des lieux, déstructuration des complémentarités fonctionnelles locales, appauvrissement des relations sociales de proximité. Ce type de constat a été fait pour quantité d'autres contextes géographiques, dans les centres-villes et les zones commerciales notamment (Mangin 2004). Toutefois, l'enjeu apparaît particulièrement important dans les régions touristiques des Alpes dans la mesure où la culture touristique et les processus sociaux d'auto-identification (Poche 1996) valorisent depuis longtemps les idées de cohésion sociale et de singularité locale nées d'un rapport particulier avec l'environnement, quitte à ce que ces représentations prennent à l'occasion la forme de stéréotypes. Pour ces raisons, les sociétés rurales alpines attachent une importance particulière à l'expression de leur singularité quand elles ont conscience que cette dernière constitue une ressource symbolique nécessaire à leur identité collective et une ressource économique, nécessaire au développement touristique.

Dans ce contexte, les initiatives portées localement pour souligner, mettre en scène, voire travailler ces singularités et réguler les flux de circulation expriment un souci de territorialiser ces flux, de les ancrer dans les lieux. Toutefois, si l'objectif est commun, les modalités de mise en œuvre sont variées. On différenciera ici quatre stratégies différentes : celles qui visent à construire l'image de la singularité des lieux, celles qui 
ambitionnent de recréer des systèmes locaux d'échange de biens, celles qui cherchent à travailler les liens sociaux, et enfin celles qui puisent dans les ressources disponibles à l'extérieur pour alimenter ce travail d'ancrage. Ces stratégies d'ancrage peuvent souvent être adoptées isolément les unes des autres. Dans les faits, elles sont souvent combinées dans de véritables projets de territoire, comme le montrent les cas des petites régions alpines étudiées dans cet article.

- Le Val d'Hérens ${ }^{2}$ est une vallée latérale du canton du Valais (Suisse). Il compte sept communes dont les trajectoires en matière de développement sont très différentes. Certaines ont connu un tourisme très ancien mais peu intensif (Evolène, Arolla); d'autres, notamment avec la station de Thyon, se sont essayées au tourisme de sports d'hiver (Mayoraz 2003). D'autres communes encore, comme Saint-Martin, bien moins touristiques, conjuguent depuis trente ans déclin économique et vieillissement de leur population. Dans l'ensemble, la faiblesse du développement touristique du Val d'Hérens contraste avec le haut niveau d'infrastructures des stations des vallées voisines (Verbier et Zermatt). Par ailleurs, comme la vallée débouche sur la ville de Sion, chef-lieu du canton du Valais, ces mêmes communes voient augmenter rapidement la proportion de leurs résidents travaillant en journée à l'extérieur.

- Le Grosses Walsertal ${ }^{3}$ est une vallée périphérique de la province du Voralberg (Autriche), une des régions alpines les plus dynamiques d'un point de vue économique et démographique, grâce notamment à son excellente accessibilité au croisement d'axes de communication majeurs qui relient l'Allemagne à l'Italie et l'Autriche à la Suisse. Le tourisme dans la province est ancien et prospère, en particulier dans un nombre considérable de stations, grandes et petites, dont la qualité des services a été dernièrement soulignée ${ }^{4}$. Parmi ces vallées touristiques, le Grosses Walsertal se distingue par une stratégie ambitieuse de développement régional et environnemental (Schermer 2002) autour de la création d'une « Réserve de biosphère » labellisée par l'UNESCO ${ }^{5}$.

- $\quad$ Chamonix $^{6}$ est la commune de Haute-Savoie (France) dont le développement touristique est à la fois le plus ancien (sa notoriété est acquise avec la visite des premiers voyageurs anglais en 1741 et l'ascension du Mont Blanc en 1786) et le plus intense : on dénombre environ 65000 lits touristiques et près de 5 millions de visiteurs par $\mathrm{an}^{7}$.

2. Les stratégies de développement dans cette vallée ont été plus particulièrement étudiées par Mathieu Petite dans le cadre d'une recherche doctorale. Les entretiens ont été conduits entre 2005 et 2007.

3. Cette étude de cas s'appuie sur des entretiens réalisés par Cristina Del Biaggio durant l'été 2007 auprès de maires de quatre communes (Raggal, Thüringerberg, Sonntag et St. Gerold) et des responsables de la réserve et du réseau de communes alpines Alliance dans les Alpes.

4. Une étude récente d'un institut de recherche suisse, le BAK Basel Economics, classe plusieurs stations du Voralberg parmi les meilleures des Alpes de ce point de vue (Kämpf et Hunziker 2008).

5. On trouvera une présentation de la Réserve de biosphère Grosses Walsertal par sa manager, Madame Birgit Reutz-Hornsteiner, à l'adresse internet suivante : www.unesco.org/mab/news/reutz_MB.pdf

6. Les stratégies de développement touristique à Chamonix ont été étudiées et suivies par Bernard Debarbieux depuis 1986 (Debarbieux 2001). Les transformations sociales récentes ont fait l'objet d'études complémentaires à la demande de la mairie et de l'office du tourisme. Les rapports d'études sont disponibles sur le site internet suivant : www.sommets-tourisme.org

7. Statistiques fournies par l'observatoire communal du tourisme, Ville de Chamonix. 
Ce développement, longtemps resté aux mains des familles locales, a pris de l'ampleur avec l'arrivée de capitaux suisses et français dès la fin du $19^{\text {e }}$ siècle (Debarbieux 2001). Il a suscité une immigration massive - la commune compte désormais près de 10000 habitants - et, de fait, un fort brassage des populations.

\section{Ancrer les flux d'images et travailler la singularité des lieux}

Le risque de banalisation des sites et des expériences touristiques est bien connu (MIT 2002). L'intense circulation des images et des modèles de pratiques et d'aménagement menace de ramener ces sites et ces expériences à de simples occurrences de formes typifiées. Les professionnels de la communication touristique, en charge de promouvoir des lieux et non des pratiques génériques, ainsi que de nombreux habitants des régions concernées, ont pris la mesure de ce risque depuis longtemps. Ils ont alors cherché à développer des initiatives visant à promouvoir des singularités locales susceptibles de spécifier l'expérience touristique. Ces stratégies ont misé sur les spécificités du lieu lui-même, promues au statut de marqueurs touristiques (tel sommet, tel site naturel, tel monument, etc.), mais aussi sur les spécificités des liens, notamment patrimoniaux, que touristes et résidents entretiennent avec lui.

À Chamonix, les professionnels du tourisme ont pris conscience du risque de banalisation du site au cours des années 1970, période d'intense urbanisation et d'équipement des domaines skiables. Ce développement a notamment pris la forme d'une standardisation des formes architecturales et de la communication touristique de la station. En réaction, depuis le milieu des années 1990, la municipalité et l'office du tourisme, souvent en partenariat avec des associations locales, ont entrepris d'affirmer la singularité du lieu de plusieurs façons : en réorientant la communication touristique de la station vers la haute montagne (paysages glaciaires, sports extrêmes, le Mont Blanc, etc.) par exemple, ou bien en multipliant les initiatives visant à rappeler le passé touristique de la station et ses spécificités (notamment à travers des expositions de documents d'archives et de photographies anciennes et la création d'un Festival des Sciences exploitant le couplage très ancien entre activités touristiques et scientifiques dans la vallée). La commune a aussi veillé à ce que le nom de Chamonix soit protégé et puisse être utilisé comme une marque, ceci afin d'éviter des utilisations contradictoires en termes d'image.

Une stratégie de singularisation par la communication et la labellisation a aussi été retenue dans le Grosses Walsertal. La création de la Réserve de biosphère a incité les acteurs locaux à adopter une démarche participative. Cette dernière a conduit à l'adoption d'un modèle (Leitbild $)^{8}$ et de plusieurs réalisations visant à spécifier le lieu : un logo, une structure unique d'information et de communication, des labels pour une série de produits agricoles et artisanaux, une stratégie d'image autour de politiques environnementales locales (transport collectif, constructions écologiques, énergie solaire).

Dans le val d'Hérens, la commune de Saint-Martin a procédé à des choix différents pour faire ressortir sa spécificité. En 1982, l'Office Fédéral (suisse) des Transports refuse

8. Ce Leitbild résulte d'un processus participatif engagé en 2004 à la faveur duquel la population a été appelée à définir des priorités et des objectifs concrets à atteindre dans les cinq ans. Le Leitbild peut être consulté à la page: www.grosseswalsertal.at/emsp/LeitbildundLogo/tabid/89/language/de-AT/Default.aspx (site consulté le 16 mai 2008). La plupart des initiatives prises dans le Grosses Walsertal dont cet article rend compte plus loin sont également présentées sur le site internet de la région. 
d'accorder une concession pour construire des remontées mécaniques sur la commune en raison d'une rentabilité estimée trop faible. En 1989, les autorités municipales renoncent définitivement au tourisme d'hiver, décision entérinée par un vote populaire, et réorientent leur stratégie sur le développement rural et la valorisation touristique du patrimoine local. Les investissements sont alors concentrés sur les sites traditionnellement investis par l'économie agropastorale, sur des spécialités locales avec notamment le label «Viande d'Hérens » qui vise à tirer profit de la notoriété d'une race bovine devenue emblématique du Valais dans son ensemble, et sur la réhabilitation des bâtiments existants. Aux yeux des porteurs de cette stratégie, ce choix permettait, mieux que l'aménagement de domaines skiables, de spécifier le développement touristique local :

"On regarde d'abord ce qu'on a, et avant de développer ce que font déjà les autres, on doit se poser la question de qu'est-ce qu'on a qui nous est spécifique. Et qu'est-ce qu'on peut faire d'innovateur? C'était ça. C'était un peu la protection du patrimoine et dire, ça c'est une chose qui a existé à l'époque. Parce que des forêts il $y$ en a partout, des montagnes, il y en a partout, des pâturages, il y en a aussi dans beaucoup d'endroits, donc ce n'est pas avec ça qu'on peut être innovateur. Mais l'idée de faire un développement en mettant en valeur le patrimoine, c'est-à-dire dans notre réflexion en gardant présent à l'esprit que pendant un certain nombre de siècles on avait vécu comme cela, et ça on aimerait le conserver et le montrer [...] on doit le mettre en valeur, parce que notre patrimoine à nous on le trouve pas nécessairement trois vallées à côté. Tandis qu'un téléski on peut le trouver dans chaque vallée, il peut être le même »".

Dans les trois contextes étudiés, il y a donc bien une stratégie explicite d'affirmation de la singularité locale, à travers des campagnes de communication, sans pourtant qu'aucune d'entre elles ne paraisse ni très originale, ni très spectaculaire.

\section{Ancrer les flux de biens et d'argent et travailler les systèmes d'échanges locaux}

La circulation des biens matériels et de l'argent est elle aussi porteuse d'un risque de standardisation, mais aussi et surtout d'un risque d'affaiblissement des systèmes économiques locaux. La prise de conscience de ce problème a suscité plusieurs types d'initiatives.

L'une d'entre elles, devenue courante dans les pays germaniques, a été adoptée dans le Voralberg dans son ensemble. Il s'agit de la création d'une «monnaie communautaire », le Talent, qui remplace l'euro dans les transactions entre des acteurs économiques volontaires. Ce dispositif vise généralement à amplifier la part proprement locale des échanges en rendant visibles les transactions qui concernent les productions et les services locaux (Blanc 2006; Schröder 2006). Au Voralberg, on met surtout l'accent sur la plusvalue économique et les liens symboliques entre habitants et produits locaux : «Avec l'achat de ces Talents vous promouvez le développement soutenable et la valeur ajoutée du Vorarlberg, parce que le Talent unit les services et produits régionaux avec les clientes et clients de la région $»^{10}$.

9. Entretien du 11 décembre 2006 avec l'ancien président de la commune de Saint-Martin.

10. www.grosseswalsertal.at/emsp/Produkte/Gutscheine/tabid/274/language/de-AT/Default.aspx 
Un autre type d'initiative, plus connu des francophones, réside dans la promotion de produits locaux, commercialisés de façon à mettre en évidence la plus-value conférée par le lieu et les conditions locales de production (par exemple les Appellations d'Origine Contrôlée). En tant que telles, ces appellations (Viande d'Hérens) sont des labels et participent plutôt, on l'a déjà dit, de stratégie de singularisation par l'image. Toutefois, le processus de spécification et de labellisation de ces productions conduit généralement à l'incorporation d'une proportion accrue d'intrants locaux, au nom de la valorisation des qualités du terroir et des savoir-faire. Au Grosses Walsertal, une gamme de produits locaux, de fabrication tantôt ancienne, tantôt récente - le fromage Walserstolz, un thé de montagne, des produits à base d'herbes et plantes autochtones - a été conçue dans le but clairement affiché de tirer un profit économique accru des ressources locales.

Un troisième type d'initiative, bien connu lui aussi, réside dans le développement de circuits courts de commercialisation qui permettent d'impliquer divers partenaires de l'économie locale et d'accroître la part des flux financiers circulant entre ces partenaires. Ce raisonnement est pour partie à l'origine de la stratégie de développement de SaintMartin en Valais et, plus généralement, de la politique agricole de la Confédération helvétique. Tout en misant sur la valorisation de son patrimoine architectural agropastoral, la commune valaisanne a cherché à coupler les activités agricole et touristique dans leur fonctionnement économique même. Le projet le plus significatif de cette stratégie a consisté à réinvestir les hameaux d'Ossona et Gréféric, abandonnés à la fin des années 1960, d'y installer une exploitation agricole et de transformer les anciens bâtiments en gîtes d'accueil touristique. Ce projet a reçu l'aide financière de l'Office fédéral de l'Agriculture en tant que projet pilote de développement rural régional tout en étant replacé - telle était la condition exigée par l'Office - dans une stratégie étendue à l'ensemble du Val d'Hérens de valorisation touristique des productions agricoles et d'agritourisme. Le même souci de mise en relation de différents acteurs a été à la base de la création du projet « Genusspechte » (pacte de la saveur) dans la vallée du Grosses Walsertal, dont le but était l'intensification des liens entre paysans et touristes. Les restaurants de la vallée qui participent à ce projet sont ainsi obligés de proposer une carte avec une proportion préétablie de produits locaux.

\section{Ancrer le lien social et travailler le commun}

La circulation des personnes a des implications d'une toute autre nature et suscite des stratégies très différentes. Ces dernières sont peut-être moins analysées que les précédentes par la littérature académique; aussi cet article leur consacre-t-il davantage de place. Ce n'est pas tant ici la cohabitation de touristes de passage et d'habitants du lieu qui justifie une analyse spécifique. Tant que la référence au lieu et l'idée que chacun se fait de sa juste place dans ce lieu sont clairement différenciées, les enjeux socio-territoriaux de l'activité touristique restent modérés. Par contre, dans un nombre croissant de situations, les rôles sociaux et les représentations correspondantes sont bien plus diversifiés et, de ce fait, plus complexes à gérer.

Les résidents secondaires, nombreux dans la plupart des régions alpines, parfois tentés par la multi-résidentialité, voire par l'installation « à demeure » quand leurs activités et l'accessibilité du lieu le permettent, tendent à développer des pratiques d'habitants. Par ailleurs, de nombreux prestataires de services touristiques, salariés ou indépendants, saisonniers ou non, privilégient des stratégies de valorisation économique du lieu 
de leur installation au détriment parfois de la qualité des interactions sociales et de la contribution à des projets collectifs. Dès lors, dans les cas extrêmes des grandes stations touristiques fortement marquées par la saisonnalité des activités, comme en Tarentaise (Savoie, France) par exemple, la question de savoir s’il subsiste une "société locale » peut être posée. Conscientes de ces transformations et soucieuses d'influer sur elles, de nombreuses communes alpines ont adopté des stratégies visant à intensifier les relations sociales entre les divers habitants et usagers des lieux et à en diversifier la nature : soutien aux commerces banals de proximité et à la vie associative, promotion d'animations culturelles et sportives et des fêtes locales, démarches participatives portant sur des enjeux de gestion ou d'aménagement, etc.

Dans le Grosses Walsertal, la création de la Réserve de la biosphère, intervenue après des décennies de déclin démographique et économique, a été justifiée dès l'origine par un double souci de développement régional et de maintien d'une vie sociale et culturelle (Lange 2005). Quelques années plus tard, les élus locaux se félicitent des échanges et de l'élargissement du cadre de réflexion que suscite la Réserve :

«On a une réunion tous les mois (Biosphärenparkkuratorium), où les 6 maires se rencontrent. Nous nous rencontrons, et c'est une bonne chose.... Au début [...] c'était contraignant pour moi de se rencontrer tous les mois ! [...] [Mais désormais] j'apprécie beaucoup le fait qu'on se rencontre tous les mois, si on a des problèmes, on peut discuter $»^{11}$.

Dans la foulée de la création de la Réserve, l'adoption de stratégies environnementales, dont on a dit qu'elles participaient d'une stratégie d'image, a aussi eu pour effet, et peutêtre aussi pour motif, de réinventer des formes de sociabilité : un réseau de femmes, «Alchemilla », s'est constitué pour valoriser les qualités gastronomiques et médicinales des plantes de montagne. La mise en place de circuits courts de commercialisation a également été justifiée par le souci de multiplier les liens et les complémentarités entre producteurs, commerçants, restaurateurs et consommateurs. Le lien intergénérationnel a été valorisé et travaillé en tant que tel dans le cadre d'un autre projet baptisé Lebensfreude (Joie de vivre), qui s'est donné pour but de promouvoir les activités collectives et proposer « une assistance optimale, humaine et individuelle » aux personnes âgées.

Dans la commune d'Evolène, dans la partie amont du Val d'Hérens, une manifestation baptisée Célébrations Interculturelles de la Montagne (CIME) semble révélatrice de plusieurs stratégies d'ancrage combinées. Organisée depuis 2006, elle permet à des groupes de musiciens et de danseurs « issus exclusivement de régions montagneuses du monde $»^{12}$ de se produire en public. À cette occasion, les artisans et les propriétaires de maisons remarquables ouvrent les portes de leur domicile et de leur atelier. Tous ensemble, les acteurs de cet événement contribuent donc à un processus de singularisation des ressources locales, par la valorisation du patrimoine culturel, et participent donc d'une opération de marketing touristique : Evolène «a su conserver son patrimoine architectural, son patois franco-provençal, le port d'un costume authentique, une agriculture de montagne

11. Entretien en allemand du 13 juin 2007 avec le maire de Raggal. Traduction de Cristina del Biaggio.

12. CIME-Célébrations interculturelles de la montagne Evolène, www.cime-evolene.ch, site internet de la manifestation (consulté le 14 avril 2008). 
vivante et des infrastructures touristiques modérées ${ }^{13}$. En cela, l'événement prolonge des initiatives locales antérieures, proches aussi de nombreuses expériences conduites dans d'autres contextes, comme les sentiers de découverte du patrimoine bâti et le musée de la vie locale créés à l'initiative de l'association culturelle Evolén'Art dans les années 1990. Mais des interviews conduites auprès des organisateurs montrent que la manifestation vise aussi la population locale avec le souci de construire un attachement des plus jeunes et des nouveaux-venus au patrimoine, aux « traditions » et au caractère « montagnard » de la culture locale : « montrer [des traditions] à l'extérieur, à des gens de passages, à des autres Valaisans, mais c'est aussi une manière de redécouvrir son propre patrimoine $»^{14}$. Sans être explicitement conceptualisée de la sorte par nos informateurs locaux, cette manifestation s'apparente donc aussi à une opération d'ingénierie identitaire. L'un des organisateurs explique cette initiative par le souci de "faire quelque chose de sympathique pour la population évolénarde, pour qu'elle se retrouve derrière un projet fédérateur $\gg^{15}$.

Dans la commune voisine de Saint-Martin, la stratégie de promotion du patrimoine bâti a cherché elle aussi, au-delà des effets déjà mentionnés en termes d'image et de complémentarité entre les activités économiques, à faire travailler la mémoire locale et les continuités symboliques des pratiques et des usages :

"La stratégie est simple, on n'a absolument rien inventé. Ça vise essentiellement à la sauvegarde du patrimoine, c'est-à-dire reconstruire ce qui a été fait il y a quelques générations en arrière. Il y a eu une prise de conscience politique: si notre génération ne réagissait pas, il y allait avoir tout un patrimoine qui allait disparaître. [...] ce patrimoine pour être construit il a fallu que plusieurs générations s'impliquent, et nous, si on ne réagit pas, il disparaît, alors on s'est dit qu'on devait à tout prix faire quelque chose $»^{16}$.

De plus, dans le projet de réhabilitation du plateau d'Ossona et Gréféric, les autorités communales ont cherché, malgré l'éloignement du site des principaux villages, à impliquer la population locale dans la conduite du projet : remise en état d'une vigne qui sera entretenue par une association de retraités, organisation régulière de manifestations populaires ponctuant l'avancement du projet, etc.

À Chamonix, les initiatives visant à créer du lien entre les habitants et les usagers sont plus originales encore. À vrai dire, le défi était d'une autre ampleur et d'une autre nature. L'attrait du lieu a suscité une fréquentation touristique, une installation de résidents secondaires et une immigration de travail et résidentielle très importantes, cela depuis des décennies. Dans le courant des années 1990, l'installation de plusieurs centaines de citoyens britanniques - environ $15 \%$ des résidents selon une estimation récente de l'administration française ${ }^{17}-a$ cristallisé l'attention et les tensions. Attirés pour certains par la reprise d'activités économiques sur place (notamment dans l'hôtellerie et la restauration) et, pour

13. www.cime-evolene.ch, idem.

14. Entretien du 15 mai 2007 avec l'un des organisateurs de CIME.

15. Idem.

16. Entretien du 30 septembre 2005 avec le président de la commune de Saint-Martin.

17. La déclaration de résidence n'étant pas obligatoire en France, cette estimation a été communiquée par les Renseignements Généraux à la mairie de Chamonix en 2007. 
d'autres, par la possibilité de résider sur place tout en travaillant pour partie à distance dans des entreprises londoniennes ${ }^{18}$, ces nouveaux habitants ont été perçus comme mal intégrés et peu soucieux d'intégration. D'autres attitudes ont fait l'objet de discussions publiques : agitation et bruit provoqués par de jeunes touristes adeptes du surf et de la vie nocturne; résidents secondaires supposés n'être motivés que par la défense de leur intérêt personnel; commerçants récemment installés accusés de ne rechercher que le seul profit financier, etc. La municipalité, inquiète de la multiplication des incidents, s'est alors engagée dans une réflexion stratégique sur le devenir de la vallée. Avec l'aide de l'office du tourisme, elle a organisé des réunions ouvertes à tous les habitants et aux professionnels du tourisme pour identifier les «valeurs » susceptibles d'être partagées par le plus grand nombre. Il a été convenu alors de mettre en œuvre deux stratégies.

La première stratégie, visant autant les touristes que les habitants et les résidents secondaires, a consisté à mettre l'accent sur le caractère exceptionnel du site, du Mont Blanc et de la haute montagne en particulier; ce faisant, la politique de communication mentionnée plus haut dans ce texte, cherchant à travailler l'image singulière du lieu, participait aussi d'une réflexion sur la construction d'attachements, sinon d'identités et de sentiment d'appartenance, partagés par ceux entre lesquels il s'agissait de construire du lien social.

La seconde de ces stratégies, plus spécialement adressée aux résidents, a consisté à sélectionner quatre " valeurs » puisées dans l'imaginaire de l'alpinisme dont il était convenu qu'elles puissent imprégner les relations sociales. Ces valeurs - « le respect, la liberté, le dépassement de soi et l'ouverture d'esprit »-ont fait l'objet d'une importante campagne de communication interne, notamment à destination des professionnels du tourisme. La démarche peut paraître aussi ambitieuse que dérisoire, comme s'il suffisait de sélectionner des valeurs et de communiquer autour d'elles pour infléchir la nature des relations sociales entre les personnes concernées. Toutefois, on ne la retient pas ici pour les effets qui sont les siens, que personne n'a entrepris de mesurer si tant est que ce soit possible, mais pour ce qu'elle nous apprend des préoccupations des élites et des sociétés locales dans un contexte touristique d'intense brassage des populations. Elle illustre une façon de concevoir le réinvestissement de la singularité d'un site et des valeurs associées à une pratique emblématique.

Dans ce cas précis, l'association entre valeurs chamoniardes et valeurs de l'alpinisme ne repose pas seulement sur la mise en scène d'une pratique sportive et touristique, si exceptionnelle soit-elle. Elle se rapporte aussi explicitement à l'histoire de la population chamoniarde elle-même et aux références identitaires dont elle s'est elle-même dotée grâce à la notoriété acquise par son environnement paysager et ses guides de haute montagne. Vouloir construire du lien social entre des individus aux trajectoires très hétérogènes en mobilisant les valeurs de l'alpinisme n'est donc pas, dans le cas si singulier de Chamonix, une concession faite aux références touristiques. L'opération est conçue, et communiquée d'ailleurs, comme garantissant une certaine forme de continuité dans l'attachement à une pratique emblématique du lien que la population locale entretient, symboliquement plus pratiquement, avec le lieu.

18. L'aéroport de Genève est à moins d'une heure et la desserte fréquente de Londres, notamment par une compagnie à bas coût, rend ce choix de résidence financièrement accessible. 


\section{Stratégies d'ancrage et ouvertures sur l'extérieur}

Présentées en contrepoint des effets perçus comme problématiques de la montée en puissance des flux liés à l'activité touristique, ces diverses stratégies d'ancrage ne doivent pas être comprises comme autant de signes de repli sur le local et de défiance à l'égard de l'extérieur. En effet, elles visent à accompagner l'activité touristique, voire à contribuer à son développement et à celui des flux qui lui sont associés, tout en les régulant. Ces stratégies s'appuient elles aussi sur des réseaux sociaux et institutionnels variés qui s'inscrivent dans des échelles plus vastes et qui leur donnent accès à des ressources mobilisables dans ce cadre.

Ces réseaux et ressources extérieurs sont de nature très variée. Il y a tout d'abord bien sûr les réseaux professionnels et de l'administration publique dans le cadre desquels certains projets, comme la stratégie de développement agro-touristique de Saint-Martin, prennent corps. Mais il ne faut pas oublier pour autant les réseaux horizontaux destinés à faciliter les échanges d'informations et d'expériences en rapport avec le type de stratégie à construire, et notamment ceux qui s'appuient sur des références alpines et montagnardes présentées comme étant communes.

Le Grosses Walsertal est très intéressant de ce point de vue. L'association des communes est membre de l'Alliance du Climat, regroupant 1400 communes et districts européens, principalement en Autriche et en Allemagne, qui partagent le souhait de développer des stratégies d'économie d'énergie dans plusieurs domaines (mobilité, construction, etc.). Par ailleurs, la Réserve en tant que telle participe activement aux activités d'un réseau alpin des espaces protégés, Alparc, dont le but principal est de faciliter les échanges d'expériences. Plusieurs projets ont bénéficié de financements Interreg. D’autres encore, comme Alchemilla, ont tiré avantage des initiatives transnationales visant à promouvoir le développement local durable dans les Alpes ${ }^{19}$. Enfin, les communes ont adhéré ensemble à l'association Alliance dans les Alpes, réseau de municipalités soucieuses de promouvoir un développement durable à l'échelle de la chaîne et de mettre en œuvre au niveau local les principes de la Convention Alpine ${ }^{20}$. Les personnes interrogées sur place ont souligné les bénéfices de ce réseau en terme d'échanges d'expériences : "Pour moi c'est déjà une grande aide de savoir qu'il y a des communes qui doivent aussi se battre sur les mêmes enjeux; cela m'apporte déjà beaucoup. Plutôt que de penser 'Je suis seul, je suis le seul à avoir ce problème avec la forêt de protection là haut'. Et cela m'apporte beaucoup $»^{21}$. À une autre échelle, des liens ont été noués avec des populations plus lointaines, en l'occurrence avec une Réserve de biosphère colombienne, afin de créer un circuit de commercialisation du café produit par de petits producteurs indépendants.

Dans le Val d'Hérens, la commune de Saint-Martin est aussi membre de l'association Alliance dans les Alpes. Le projet de réhabilitation des hameaux d'Ossona et Gréféric a

19. Alchemilla a été financé par le cadre du programme Dynalp2 initié par la Conférence Internationale pour la Protection des Alpes (CIPRA) et financé par la Fondation de droit suisse MAVA.

20. Ce réseau rassemble 250 communes de sept pays de l'arc alpin. Il a une activité régulière autour de séminaires, de concours de projets et de représentations dans les instances de la Convention Alpine. Pour plus d'informations : www.alpenallianz.org/fr (consulté le 20 mai 2008). La Convention Alpine est signée en 1991 par les États alpins et l'Union Européenne visant à coordonner les initiatives des États dans la promotion du développement durable.

21. Entretien en allemand du 13 juin 2007 avec le maire de Raggal. Traduction par Cristina del Biaggio. 
été retenu au titre du programme Dynalp1, initié par cette association, mais financé par le programme Interreg Espace Alpin.

Chamonix, enfin, a noué plusieurs jumelages avec d'importantes stations touristiques de par le monde. Elle est membre d'une association de grandes stations alpines - Best of the Alps - dont l'objectif principal est d'aider ses membres à organiser leur promotion commerciale sur les marchés lointains. La mairie et l'Office du tourisme organisent aussi une manifestation annuelle - les Sommets du Tourisme - qui vise à présenter et discuter des expériences de tourisme durable dans des contextes variés.

Ces communes, et les régions alpines qui illustrent dans cet article la variété des stratégies d'ancrage des flux engendrés par le tourisme, ne prônent donc aucune forme de repli, bien au contraire. Leur préoccupation principale réside dans la régulation de ces flux et pour ce faire, une circulation d'informations ciblées constitue une ressource en soi. On observe alors que les espaces de référence dans lesquels les acteurs cherchent à développer les interactions dans chacune de ces trois études de cas sont parfois aussi ceux qui servent de ressources au travail symbolique sur le lien social, pour ce qui touche aux identités collectives en particulier.

\section{Conclusion}

Aussi divers soient-ils de par la nature des lieux concernés et des initiatives étudiées, ces exemples ont en commun d'illustrer ce que l'on a proposé d'appeler ici des stratégies d'ancrage ou de territorialisation. Ces stratégies visent à répondre aux craintes suscitées par les circulations induites par l'activité touristique, ou aux effets de celle-ci tels qu'ils sont perçus dans les localités respectives.

Au-delà de ces illustrations, notre étude se veut porteuse d'une proposition générale sur l'analyse du phénomène touristique. Elle suggère que la singularité du lieu et du territoire, habituellement conçues comme étant la condition et la ressource de l'activité touristique, deviennent, une fois l'activité installée et amplement développée, un enjeu d'initiatives sociales et de politiques publiques. Ce changement de statut, du lieu-ressource au lieuprojet, du lieu-donné au lieu-produit, met en évidence la capacité de transformation des lieux dont dispose le tourisme dans l'usage qui est le sien. Par les circulations qu'il induit, de par sa nature même et les pratiques économiques et sociales qu'il engendre, le tourisme tend à défaire les relations singulières que les sociétés locales entretiennent en leur sein et avec leur environnement. Les sociétés concernées peuvent s'en satisfaire, si les individus qui les composent y trouvent leur compte d'une autre façon. Mais dans le cas contraire, le tourisme suscite parallèlement à son développement des réactions collectives qui dès lors tendent à faire du territoire et des relations sociales territorialisées un enjeu collectif et un projet.

Les modalités de ce processus de territorialisation sont variées. Elles mobilisent les ressources économiques, symboliques, politiques et identitaires des acteurs locaux. Elles agissent aussi en jouant de la pluralité des échelles : échelle locale concernée par le processus touristique lui-même; ou bien échelles régionale, nationale et supranationale pour mobiliser d'autres ressources ou d'autres images, comme dans le cas de l'identification aux Alpes et à la montagne.

\section{Remerciements}

Cet article combine des résultats de recherche obtenus dans le cadre de plusieurs projets conduits successivement ou simultanément au sein de l'équipe «Montagnes : 
Connaissances et Politiques » de l'Université de Genève. Les auteurs remercient en particulier le Fonds National de la Recherche Suisse pour avoir financé le projet «PanAlpine networks » dans le cadre duquel les terrains du Grosses Walsertal et du Val d'Hérens ont été travaillés. Ils remercient aussi la municipalité de Chamonix pour son soutien et son intérêt pour les études réalisées entre 1998 et 2004. Ils remercient enfin leurs informateurs pour leur disponibilité.

\section{Références citées}

Appadurai, Arjun, 1996. Modernity at large. Cultural dimensions of globalization. London, Minneapolis: University of Minnesota Press.

Bätzıng, Werner, 2003. Die Alpen. Geschichte und Zukunft einer europäischen Kulturlandschaft. München: C.H. Beck.

Blanc, Jérôme (dir.), 2006. Exclusion et liens financiers : Monnaies sociales. Rapport 2005-2006. Paris : Économica.

Bradshaw, Michael J., 1990. "New regional geography, foreign-area studies and Perestroika", AREA, 22 (4), pp. $315-322$.

Castells, Manuel, 1999 [1996]. L'ère de l'information. Le pouvoir de l'identité. Paris : Fayard.

Debarbieux, Bernard,

1997. «La nomination au service de la territorialisation : réflexions sur l'usage des termes 'alpe' et 'montagne' », Le Monde Alpin et Rhodanien, 2-4, pp. 227-241.

2001. Chamonix-Mont Blanc (1860-2000). Les coulisses de l'aménagement. Servoz : Edimontagne.

Featherstone, Mike, Lash Scott et Roland Robertson (eds), 1995. Global modernities. London: Sage Publications.

Gottmann, Jean, 1952. La Politique des États et leur géographie. Paris : Armand Colin.

Hannerz, Ulf, 1996. Transnational connections. London: Routledge.

HoLmÉn, Hans, 1995. "What's new and what's regional in the 'new regional geography'?", Geografiska Annaler, 77 B, pp. 47-63.

KäMPF, Richard et Christian HunzIKER, 2008. Succès et compétitivité du tourisme alpin. Bâle et Berne : BAK Basel Economics et Secrétariat d'État à l'économie (SECO).

LANGE, Sigrun, 2005. Leben in Vielfalt. UNESCO-Biosphärenreservate als Modellregionen für ein Miteinander von Mensch und Natur. Wien: Österreichische Akademie der Wissenschaften.

MangIn, David, 2004. La ville franchisée. Formes et structures de la ville contemporaine. Paris : Éditions de la Villette.

MAYORAz, Didier, 2003. Le Val d'Hérens face au défi touristique hivernal (1960-2000). Mémoire de licence en histoire contemporaine, Université de Fribourg.

Mit, 2002. Tourismes 1. Lieux communs. Paris : Belin.

Offner, Jean-Marc et Denise PumAin (éds), 1996. Réseaux et territoires : significations croisées. La Tour d'Aigues : Éditions de l'Aube.

Poche, Bernard, 1996. L'espace fragmenté. Eléments pour une analyse sociologique de la territorialité. Paris : L'Harmattan.

RafFestin, Claude, 2006. "Réflexions hétérodoxes sur la globalisation », Revue Européenne des Sciences Sociales, 44 (134), pp. 247-257.

Reichler, Claude et Roland Ruffieux, 2000. Le voyage en Suisse: anthologie des voyageurs français et européens de la Renaissance au $20^{e}$ siècle. Paris : Robert Laffont. 
Relph, Edward, 1976. Place and Placelessness. London : Pion.

SCHERmer, Markus, 2002. "The formation of 'Eco-regions' in Austria and the role of the structural fund", Centre for Mountain Agriculture, University of Innsbruck.

SCHROEDER, Rolf, 2006. "Community exchange and trading systems in Germany", International Journal of Community Currency Research, 10, pp 24-42.

TARrius, Alain, 2000. Les nouveaux cosmopolitismes : mobilités, identités, territoires. Paris : Éditions de l'Aube.

Tissot, Laurent, 2000. Naissance d'une industrie touristique : Les Anglais et la Suisse au 19e siècle. Lausanne : Payot.

TuAn, Yi-Fu, 1977. Space and place: the perspective of experience. Minneapolis: University of Minnesota Press.

URRY, John, 2005. Sociologie des mobilités. Une nouvelle frontière pour la sociologie. Paris : Armand Colin. 